\title{
Characterizations of enriched metallic single-walled carbon nanotubes in polymer composite
}

Bin Chen ${ }^{l}$, Jing $L i^{l}$, Yijiang $L u^{l}$, Martin Cinke ${ }^{l}$, Dyng $A u^{2}$, Harry Partridge and $M$. Meyyappan

Center for Nanotechnology, NASA Ames Research Center, MS 229-1, Moffett Field, CA 94035

Julie P. Harmon, Patricia Anne O. Muisener, LaNetra Clayton, John D'Angelo,

Department of Chemistry, University of South Florida, 4202 E. Fowler Avenue, Tampa, Fl 33620

Email: bchen@mail.arc.nasa.gov

Summary: Using different processing conditions, we disperse the singlewalled carbon nanotube (SWNT) into the polymethyl methacrylate (PMMA) to form composites. In the melt-blended sample, the SWNTs - originally semiconducting - became predominantly metallic after dispersion into the melt-blended composite. The interaction of the PMMA and SWNT is investigated by the polarized Raman studies. The structure changes in the PMMA and SWNT shows that the anisotropic interactions are responsible for SWNT electronic density of states (DOS) changes. The increased metallic SWNT percentage is confirmed by the conductivity and dielectric constant measurements.

Keyword: single-walled carbon nanotubes, nanotechnology, Raman spectroscopy, conductivity, PMMA composite, dielectric constant

${ }^{1}$ ELORET Corp.

${ }^{2}$ NASA summer intern from Department of Electrical Engineering, University of California at Santa Cruz 


\section{Introduction}

Nature provides us with two distinct carbon forms - diamond and graphite. Diamond is one of the hardest materials on earth, while graphite is one of the most electrical conductive non-metal materials. The recently discovered synthetic carbon form, single walled carbon nanotube (SWNT) combines both of these two distinguished properties. The excellent electronic transport property and mechanical strength, promise unprecedented applications for electronic, sensing and actuating devices and components.

As-grown SWNTs are a mixture of semiconducting and metallic nanotubes in an approximate 2:1 ratio. The separation of the SWNT mixture is further complicated by the rope-like hydrophobic aggregates consisting of semiconducting and metallic tubes, which are bundled together due to their high aspect ratio. In order to tailor the isolated SWNT with the desired charility, proper dispersion and self-assembly schemes have become recent focuses ${ }^{1}$. One of the promising approaches to break the bundles is to disperse them into a polymer matrix or forming a composite. This approach is especially attractive since the polymer processing platform can be readily adapted for many SWNT applications.

The PMMA polymer matrix used in this study has good structural stability and flexible processability. However, this class of materials is electronically insulating. The mobility needs to be increased to at least $10^{-6} \mathrm{~S} \mathrm{~m}^{-1}$ in order to be used for passive electronic components and electromagnetic shielding ${ }^{2}$. SWNT polymer composites have been proposed as a possible solution. In this report, we demonstrate that the electronic conductivity is enhanced in the thermally processed composites through anisotropic interaction between PMMA and SWNT, while the UV polymerized composites have no such anisotropic effects, resulting in no increase in its electronic properties.

As diamond and graphite yield distinct Raman signatures, the SWNT displays not only both $\mathrm{sp}^{3}$ and $\mathrm{sp}^{2}$ Raman features, but also unique characteristics in the one-dimensional nanometer scale. The SWNT is a unique form of polymer consisting of only one element - carbon. The chromophoric feature of C-C bonds in both CNT and polymers yields a very strong scattering signal, making Raman spectroscopy an ideal technique to study the interaction between the constituent components in the composites. As the Raman excitation energy becomes resonant with the SWNT van Hove Singularity (VHS) gaps in the $1 \mathrm{D}$ electronic density of states (DOS), the semiconducting or metallic samples yield distinct responses depending on the diameter and chirality. Two characteristic SWNT signatures ${ }^{3}$ include the tangential mode (TM) G-band around $1590 \mathrm{~cm}^{-1}$ and D-band around $1350 \mathrm{~cm}^{-1}$. The semiconducting nanotubes display a symmetric line shape profile around $1590 \mathrm{~cm}^{-1}$, whereas metallic nanotubes exhibit a broad asymmetric G-band around $1560 \mathrm{~cm}^{-1}$ as well as a sharp band around $1580 \mathrm{~cm}^{-1}$. The Raman TM symmetry profile of SWNT can be used to study its electronic properties. Furthermore, polarized Raman study probes the conformation changes in the composite, depending on the interfacial interactions and the orientations of the two components in the nanocomposite. The nonrandom molecular orientation can be investigated through depolarizations. 
Previous resonant Raman studies ${ }^{4}$ demonstrated that the anisotropic interaction between SWNT and PMMA is responsible for the changes in the electronic DOS of the SWNTs in the melt-blended composite. In this work, polarized Raman technique is used to study the anisotropic interactions between the PMMAs, and between PMMA and SWNT side wall.

\section{Experimental}

SWNTs produced from laser ablation techniques were purified according to the procedure in the literature 5 . PMMA-SWNT composite films were synthesized by two different techniques. In one approach, we formed a melt-blended composite by dissolving and sonicating the oven-polymerized PMMA and SWNT in N, N, dimethylformamide. This composite was dried in the oven at $275^{\circ} \mathrm{C}$, then melt-pressed above PMMA glass transition temperatures five times, to ensure uniform mixing. In the second approach, the composite was processed by in-situ UV polymerization of the methyl methacrylate (monomer) in the presence of SWNTs with an initiator in the sonication shear field. In both cases, the SWNT loading is $0.026 \%$ (wt \%). All samples were cast into films for analysis.

The Raman analysis set up has been described elsewhere ${ }^{6}$. In this study, we employed a $633 \mathrm{~nm} H \mathrm{He}$ : Ne laser to excite the composites. Polarized Raman spectra were obtained with a set of linear polarizer combined with the half waveplate for detecting the perpendicular and parallel Raman scattering respectively. The $\mathrm{CCl} 4$ is used for the calibration of the depolarization ratio: $\rho=I_{1} / / /$, where $I_{1}$ and $I_{/ /}$are the Raman scattering intensity that perpendicular and parallel to the incident light respectively. Dielectric constants were measured under nitrogen purge of $500 \mathrm{ml} / \mathrm{min}$. The frequency was swept over a range of 0.1 to $300,000 \mathrm{~Hz}$ in room temperature. The conductivity measurement was preformed with a HP4156B semiconductor parameter analyzer, equipped with a Low Temperature 4-Probe Station (LTMP-4) and a tungsten tip of $0.35 \mu \mathrm{m}$ diameter (MMR Technologies, Inc). Two microprobes in the LTMP-4 were placed to the samples about $2 \mathrm{~mm}$ apart, with a voltage swept from $-5 \mathrm{~V}$ to $+5 \mathrm{~V}$ at $20 \mathrm{mV} / \mathrm{sec}$.

\section{Results and discussions}

The SEM analysis shows good dispersion of SWNT in the PMMA matrix. As control samples, oven and UV polymerized PMMA were examined by Raman spectroscopy and their chemical structures arising from the two processes, were compared. The resultsin figure 1 show very similar vibrational symmetry and characteristic frequencies ${ }^{7}$, although the UV polymerized PMMA gives a higher fluorescence level, possibly due to the presence of monomer and other impurities in the samples.

The polarized Raman is a sensitive approach to analyze vibration symmetries of the two composite ensembles, because the most symmetric vibration modes have the lowest depolarization ratios. Figure 2a shows that the polarized Raman spectra of PMMA, meltblended and UV-polymerized composites. All of them show consistent polarized behavior in the $\mathrm{CH}_{2}$ backbone stretch and $\mathrm{C}=\mathrm{O}$ sidechain bending. The asymmetric 
backbone $\alpha-\mathrm{CH}_{3}$ stretching $\left(2953 \mathrm{~cm}^{-1}\right.$ ) is substantially enhanced for all three samples, while the symmetric $\mathrm{CH}_{2}$ stretch $\left(2942 \mathrm{~cm}^{-1}\right)$ decreases in the perpendicular scattering. Similarly, the symmetric sidechain $C^{\alpha} C^{\beta}$ and $\mathrm{CO}$ stretch and bending modes $\left(810 \mathrm{~cm}^{-1}\right)$ decreased drastically while the rocking mode of $\alpha-\mathrm{CH}_{3}\left(970 \mathrm{~cm}^{-1}\right)$ increases in the PMMA and both the composites in the same trend as shown in Figure 2b. However, the melt-blended composite (Figure $2 c$ )shows the different polarization response from the UV-polymerized composite as well as the neat PMMA. The side chain $\mathrm{C}=\mathrm{O}$ stretch and $\mathrm{CCO}$ deformation modes $\left(1725 \mathrm{~cm}^{-1}\right)$ remains unchanged in the melt-blended composite in either Raman scatterings, but decreases in the polarization perpendicular to the incident light in both UV-polymerized composite as well as neat PMMA. The different polarization responses are the results of the orientation of the PMMA and SWNT interaction in the composite. The schematic interactions can be illustrated in figure 3 . The anisotropic interaction between the SWNT sidewall and PMMA backbone shown in Figure 3a gives more spatial freedom for the PMMA side chain than that in the random ensembles of PMMA in the neat PMMA or in the UV-polymerized composite shown in figure $3 \mathrm{~b}$. Since the asymmetric vibrations, originated from more randomly orientations PMMA side chain, are less polarized, therefore, the PMMA in the melt-blended composite shows almost the same intensity in both scatterings that perpendicular and parallal to the incident light correspondingly. On the other hand, the UV polymerized composite has the same depolarization ratio as that of neat PMMA, which, is smaller than that of the PMMA in the melt-blended composite. Thus, the polarized Raman results in figure 2 are consistent with the schematic anisotropic interactions between the PMMA backbone and the SWNT side wall in the melt-blended composite (Figure 3a).

The increased metallic SWNT features was revealed by the resonant excitation energy with He:Ne laser through the G-band Breit-Wigner-Fano lineshapes analysis in the previous Raman study. This enhanced metallic conductivity in the melt-blended composite has been confirmed by dielectric constant measurements as well as conductivity measurements. Figure 4 a shows that the linear I-V curve of the PMMA, giving a constant resistance with the changes in voltage. The conductance obtained from the the slope is as low as $2.5 \times 10^{-12} \mathrm{~S}$. Figure $4 \mathrm{~b}$ shows the non-linear I-V response from the melt-blended composite with larger slope. The conductivity of melt-blended composite is increased by 3-4 orders of magnitude compared to the neat PMMA. Clearly, the metallic conductivity increase in this composite is a direct evidence that the percentage of the metallic SWNT in the mixture has increased. The curved semiconducting I-V characteristics on the positive as well as negative bias region also indicates that the semiconducting SWNT still exist in the composite. In contrast, the decreased conductivity of the UV polymerized composite over the neat PMMA is observed in Figure $4 c$. The conductivity results are consistent with table 1 , showing the dielectric constant measurements at room temperature. Interestingly, the UV polymerized composite shows the decrease of dielectric constant compared to the neat PMMA, contrary to the intuition that SWNT usually increase the composite conductivity. Based on our previous Raman results of the Raman shifts of radiant breathing modes, as well as the broadening of the G-band, we believe that the sidewall functionalization by the UV initiator can breakdown some of the sidewall $\pi$-conjugation, therefore, decrease the 
conductivity in this composite.

\section{Conclusion}

We have shown that metallic conductivity of SWNT is enhanced through SWNT melt blending with PMMA. The resultant composite exhibits semiconducting as well as metallic characteristics, in the whole bias range at the room temperature due to the conductivity path provided by increased metallic SWNT. The thermal processing is responsible for enhancing the composites electronic conductivity through anisotropic interactions between the SWNT sidewall and the PMMA backbone. This polarizability induced anisotropic interaction can be optimized for tailoring the SWNT in nanoelectronic applications.

\section{Acknowledgements}

Work by Eloret authers is supported by NASA contract no. NAS 2-99092. 
Figure 1

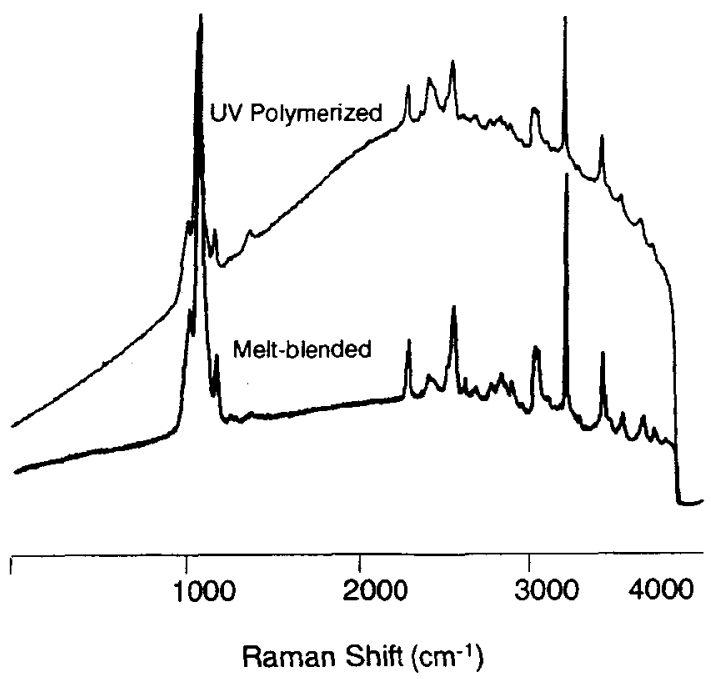


Figure 2 a

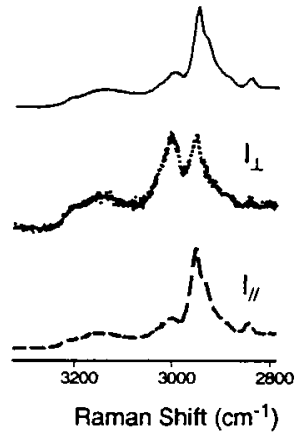

Melt-blended

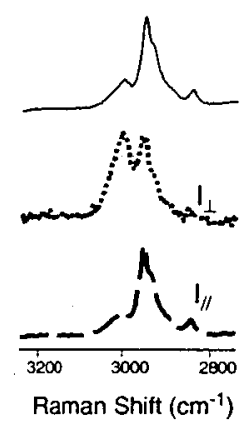

UV-polymerized

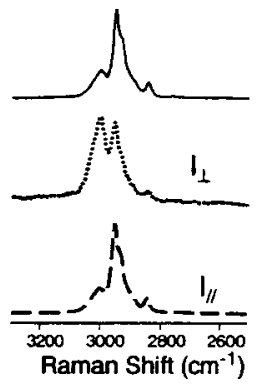

PMMA 
Figure $2 b$

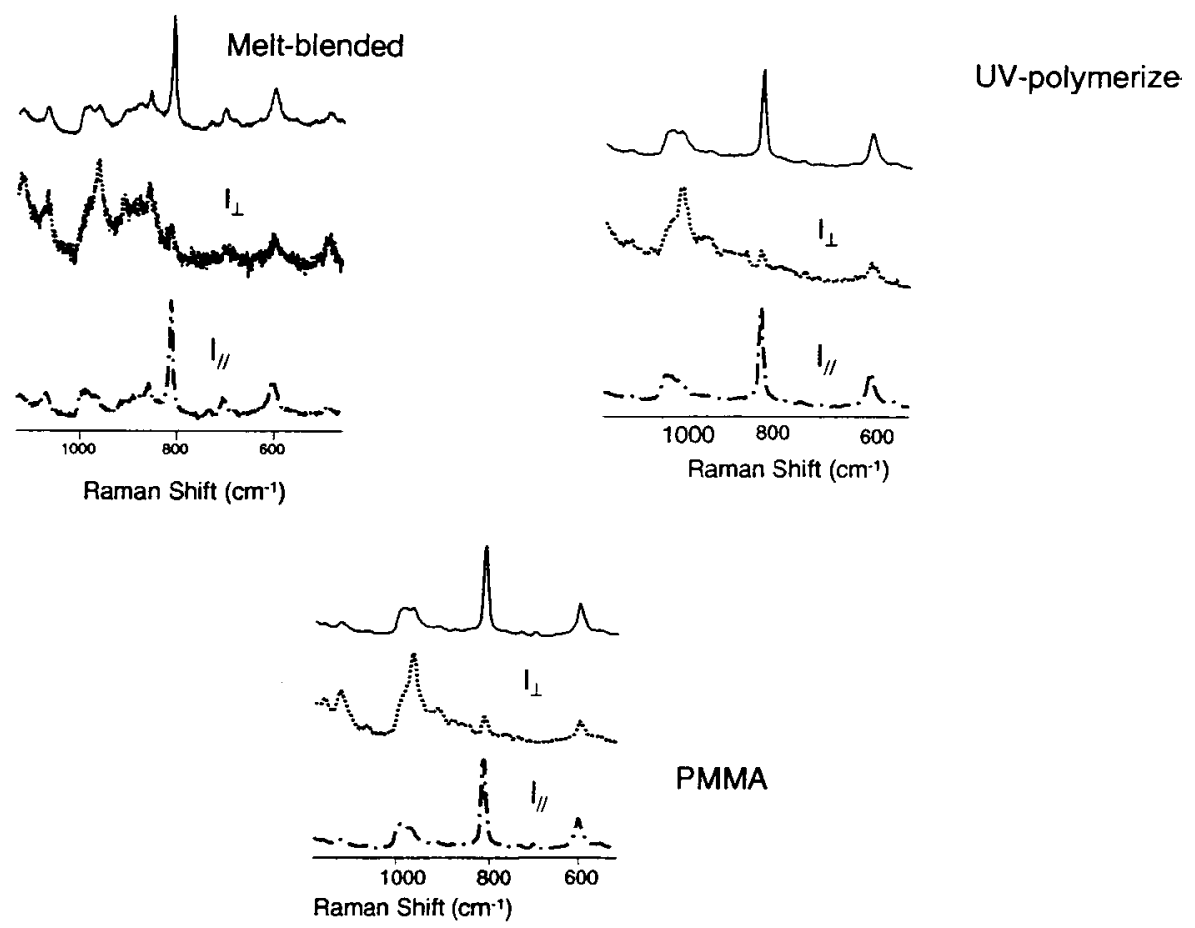


Figure 2c

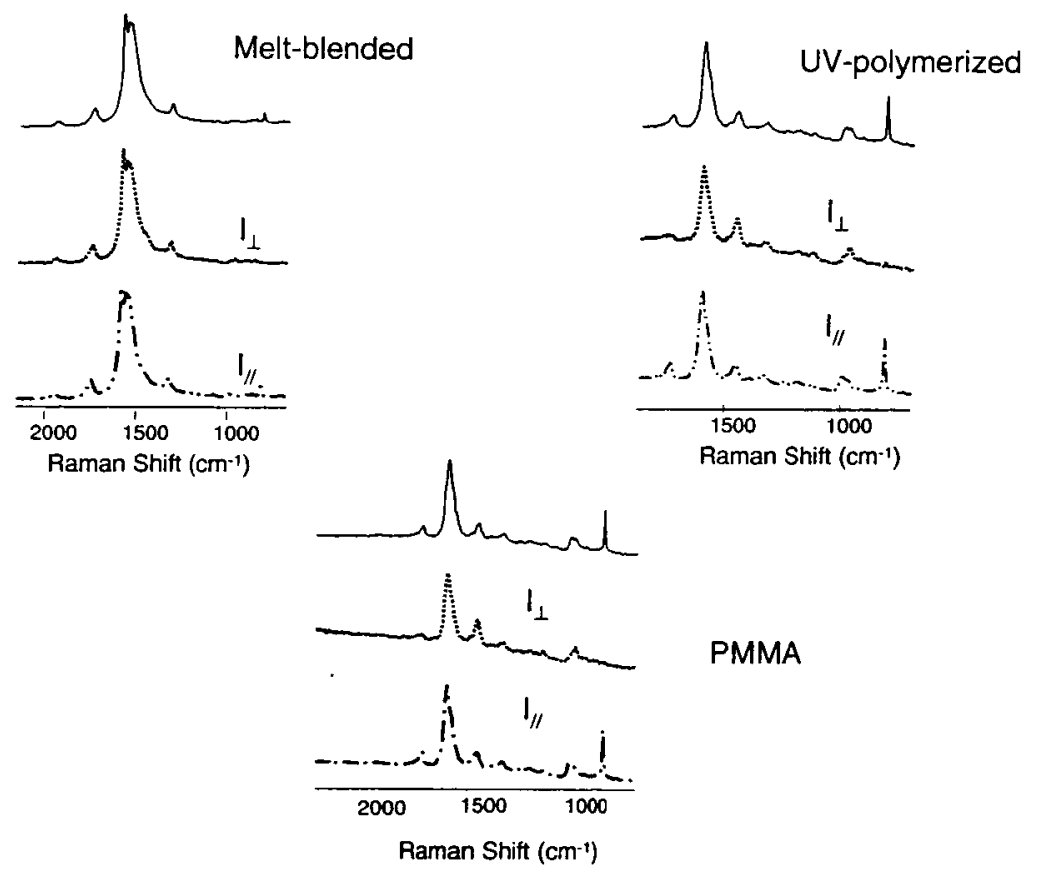


Fig. 3a

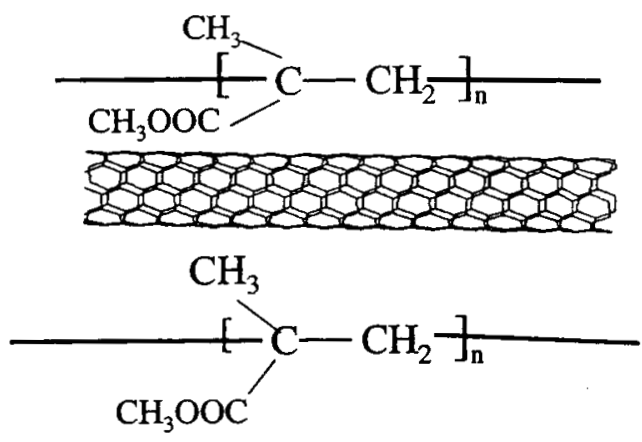




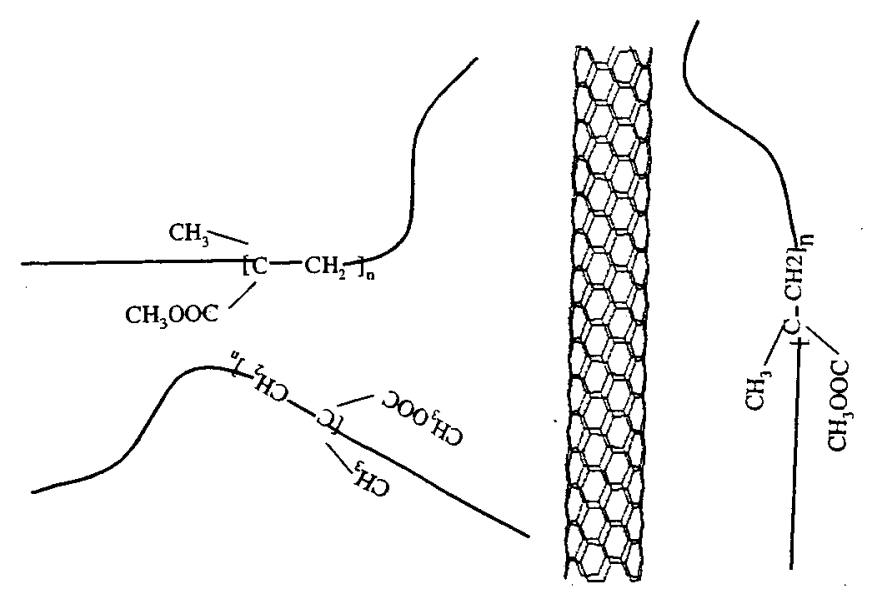

Fig. 3b 
Figure 4a

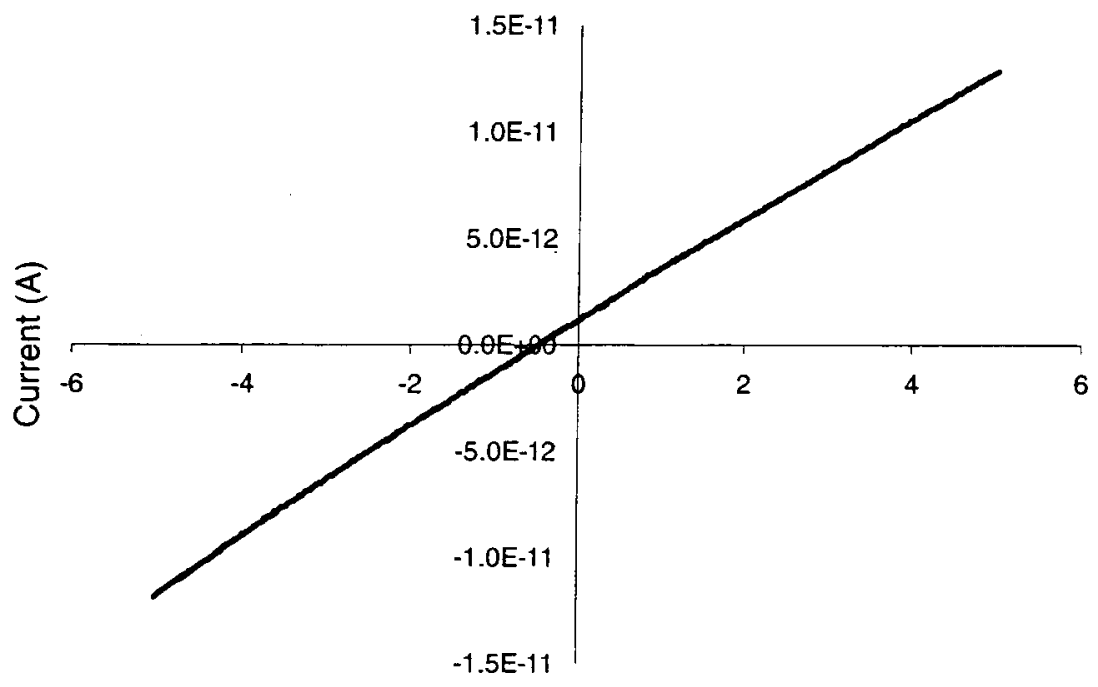

Voltage (V) 
Figure 4b

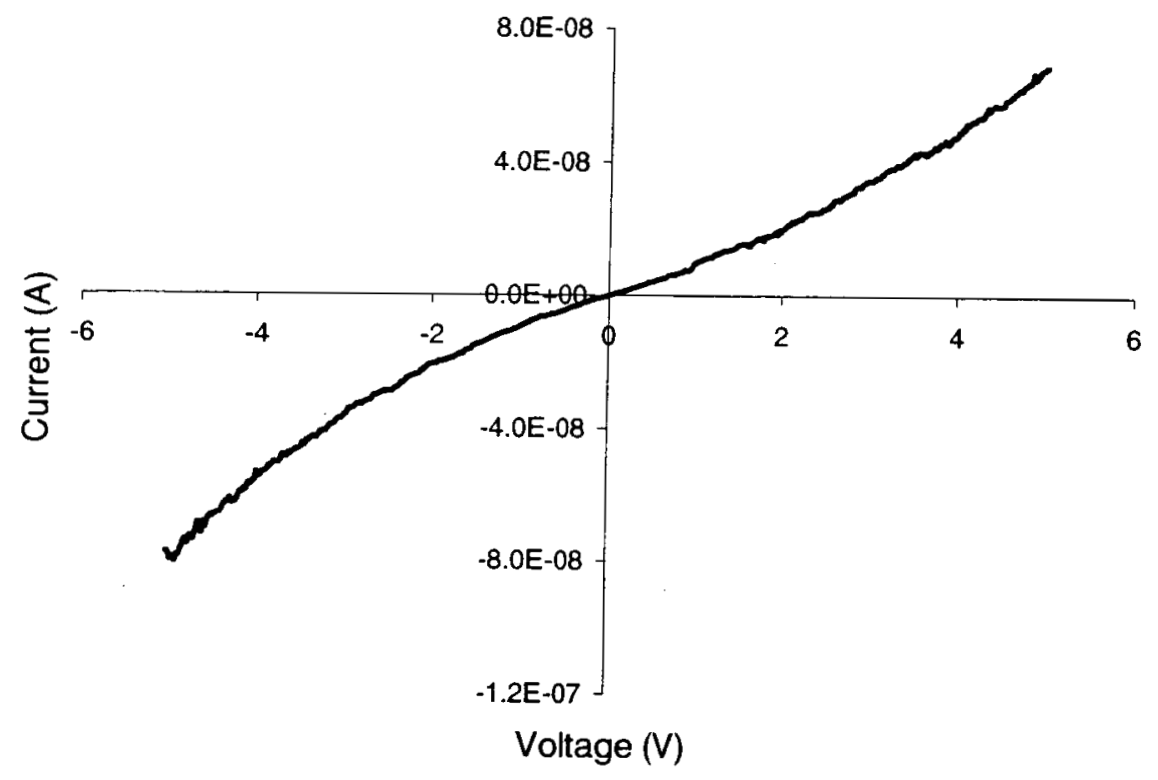


Figure $4 c$

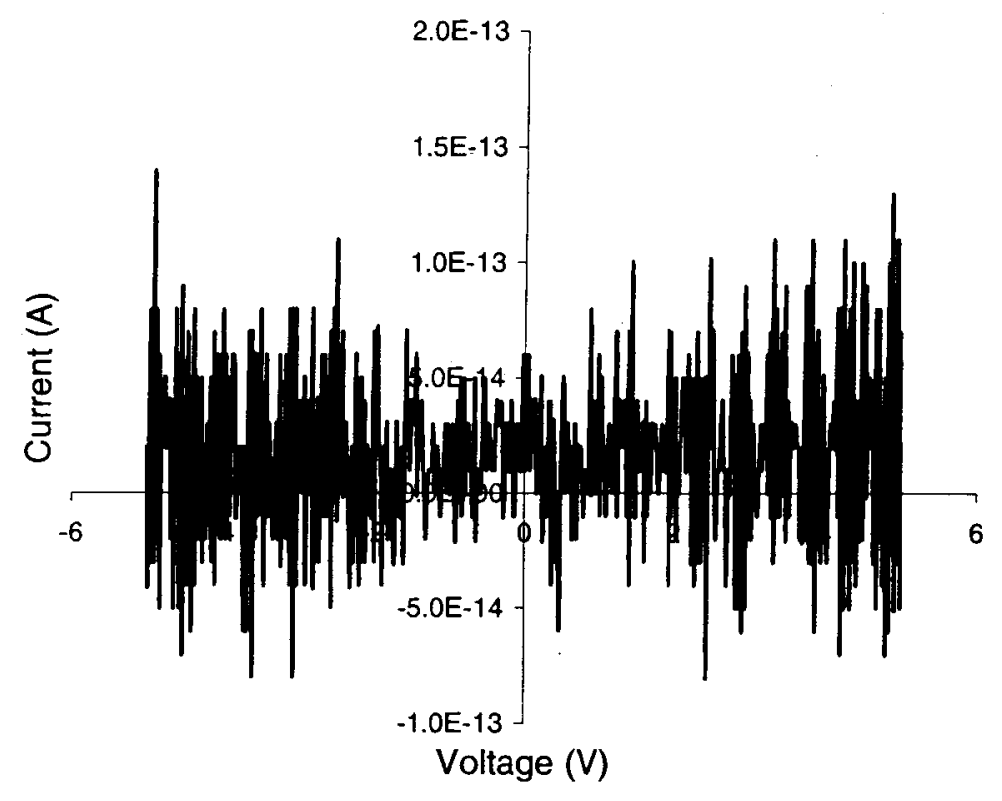


Table 1 Dielectric constants of the controlled PMMA and the composite at room temperature

\begin{tabular}{|c|c|c|}
\hline & UV polymerized & Melt blended \\
\hline Composites & 1.56 & 4.52 \\
\hline PMMA (Control) & 3.7 & 2.65 \\
\hline
\end{tabular}




\section{Figure Captions}

Figure 1. Raman spectra of the PMMA processed from UV polymerization and meltblending. The similar vibration symmetry and characteristic frequencies show the same chemical structure, though UV polymerized PMMA shows a higher fluorescence level, possibly due to the presence of monomer and other impurities in the samples.

Figure 2 Polarized Raman spectra of the PMMA and the two PMMA-SWNT composites under non-polarized scattering, perpendicular and parallel to the incident light scattering, from top to the bottom of each panel respectively.

a. $\mathrm{CH}_{2}$ backbone stretch and $\alpha-\mathrm{CH}_{3}$ stretching region. In all three samples, the asymmetric backbone $\alpha-\mathrm{CH}_{3}$ stretching $\left(2953 \mathrm{~cm}^{-1}\right)$ is substantially enhanced, while the symmetric $\mathrm{CH}_{2}$ stretch $\left(2942 \mathrm{~cm}^{-1}\right)$ decreases, in the perpendicular scattering.

b. PMMA side chain bending and rocking region. The symmetric sidechain $\mathrm{C}^{\alpha} \mathrm{C}^{\beta}$ and $\mathrm{CO}$ stretch and bending modes $\left(810 \mathrm{~cm}^{-1}\right)$ decrease drastically while the rocking mode of $\alpha$ $\mathrm{CH}_{3}\left(970 \mathrm{~cm}^{-1}\right)$ increases in the neat PMMA and both the composites in the same trend. c. The $\mathrm{C}=\mathrm{O}$ stretch and $\mathrm{C}-\mathrm{C}$ stretch region. The $\mathrm{C}=\mathrm{O}$ stretch and $\mathrm{CCO}$ deformation modes $\left(1725 \mathrm{~cm}^{-1}\right)$ remain unchanged in the melt-blended composite in either Raman scatterings, but decrease in the polarization perpendicular to the incident light in the UVpolymerized composite as well as neat PMMA.

Figure 3 Schematic illustrations of the PMMA and SWNT anisotropic and isotropic interactions, in the melted blended and UV polymerized composites respectively. a. The anisotropic interaction between SWNT sidewall and PMMA backbone shown in gives the PMMA side chain more spatial freedoms than the random ensembles of PMMA in the neat PMMA and UV-polymerized composite

b. The side chain vibrations of PMMA in the UV-polymerized composite and neat PMMA are hindered by the random orientations of the SWNT side wall to the PMMA backbone

Figure 4 . The conductivity measurements for PMMA, melt-blended and UV-polymerized PMMA/SWNT in $a, b, c$ respectively.

a. The low conductivity obtained from the slope is $2.5 \times 10^{-12} \mathrm{~S}$.

b. The non-linear I-V response from the melt-blended composite. The conductivity of melt-blended composite is increased by 3-4 orders of magnitude compared to the neat PMMA. The curved semiconducting I-V characteristics on the positive as well as negative bias region also indicate the substaintial amount of semiconducting SWNT exist in the composite.

c. The decreased conductivity of the UV polymerized composite over the neat PMMA is observed. 


\section{Reference}

${ }^{1}$ R. Krupke, F. Hennrich, H. v. Löhneysen, M. M. Kappes, Science, Vol. 301, 344 (2003).

${ }^{2}$ D. D. L. Chung, Carbon, Vol. 39, 279 (2001).

${ }^{3}$ A. M. Rao, E. Richter, S. Bandow, B. Chase, P. C. Eklund, K. A. Williams, S. Fang, K. R. Subbaswamy, M. Menon, A. Thess, R. E. Smalley, G. Dresselhaus and M. S. Dresselhaus, Science, 275, 187 (1997).

${ }^{4}$ B. Chen, M. Cinke, J. Li and M. Meyyappan, Z. Chi, J. P. Harmon, P. A. O. Muisener, L. Clayton, J. D'Angelo, submitted to Phys. Rev. B.

${ }^{5}$ J. Liu, A.G. Rinzler, H. Dai, J. H. Hafner, R.K. Bradley, P.J. Boul, A. Lu, T Inverson, K. Shelimove, C.B. Huffman, F. Rodriguez-Macias, Y-S. Shon, T.R. Lee, D.T. Colbert and R. E. Smalley, Science 280, 1253 (1998).

${ }^{6}$ B. Chen, G. Parker I, J. Han, M. Meyyappan, and A. M. Cassell, Chem. Mater., Vol. 12, 1891 (2002).

${ }^{7}$ J. Dybal and S. Krimm, Macromolecules, Vol. 23, 1301 (1990). 